\title{
Analysis of Dynamic Route Discovery Mechanism in Reactive Routing Protocols
}

\author{
Neeraj Bhargava, Ph.D \\ Associate Professor, \\ Dept. of Computer Science, \\ School of Engineering \& \\ Systems Sciences, \\ MDS University, Ajmer, India
}

\author{
Anchal Kumawat \\ Research Scholar, \\ Dept. of computer Science, \\ School of Engineering \& \\ System Sciences, \\ MDS University, Ajmer, India
}

\author{
Ritu Bhargava, Ph.D \\ Lecturer, \\ Department of MCA, \\ Govt. Women's Engineering \\ College, \\ Ajmer, India
}

\begin{abstract}
The wireless network increases the mobility of devices day by day. In this case energetic devices perform an important role. Mobile Ad-hoc network is the network which is moves dynamically and creates its own visibility. The AODV is the protocol of Mobile ad-hoc network in which it is work as reactive routing protocol. The implementation of this protocol, we have done by the NS2 simulation software. NS2 is the tool to designed dynamic network. This paper discussed about AODV protocol and its related aspects such as link break due to mobility, end-to-end delay, throughput and role of inter mediator between sender and receiver.
\end{abstract}

\section{Keywords}

Keywords: MANET, AODV, NS2, Wireless Network, Throughput.

\section{INTRODUCTION}

Mobile Ad-hoc Network (MANET) is Virtual Network or Wireless Network. It is collection of various Mobile devices that are using radio waves as a transmission medium. MANET works in transmission range. It is a Multi hop Network. In which every node work as a router. If any node wants to send data to another node and another node establish at more distance then it will uses various intermediate nodes to transmit the message. In this process including all the nodes work as router and defined Multi hop network. The performance of network slowdown, when multiple routers involving in the communication process. The direct communication between sources-to-destination increases the speed of communication. MANET network makes the dynamic topology it can be changed according to node movement. This paper addresses the routing problem and changed position of nodes in mobile ad-hoc network without considering the following issues such as QOS (Quality of Service), security and access control mechanism. [1][2][3][7]

\section{MANET has two types:}

1. Vehicular Ad-hoc Network (VANET's) used in vehicle and road side equipment.

2. Internet Based Mobile Ad hoc Network (iMANET's) used in mobile nodes.

NS-2 is the Network simulation software which is used to design multiple network models with their routing protocols. NS-2 provides various features to design netᄀwork model. The NS-2 is an event driven and object oriented network simulation tool.

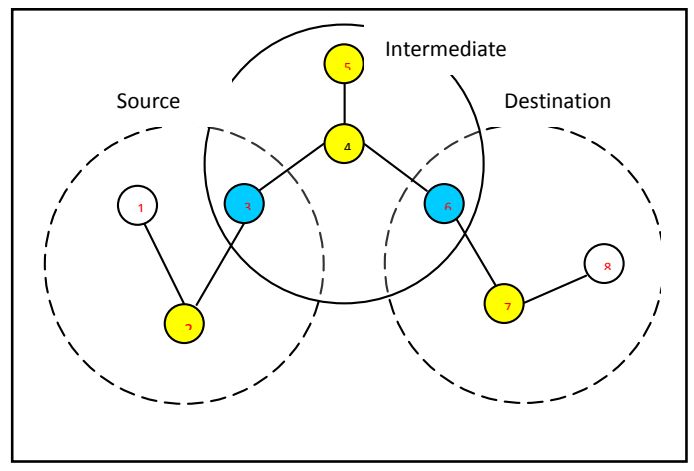

Fig 1: MANET Routing Protocol

The Main objective of MANET routing protocol is to suggest, maximize efficient energy. Nodes in MANET depend on limited energy resources. Various routing protocols of MANET's have been suggested like: DSR, AODV, DSDV, TORA and ZRP. But in this paper we have considered only AODV protocol [4].

\section{OUTLINE OF ROUTING PROTOCOLS OF MANET}

\subsection{Classification of Protocols}

There are many ways to classify the routing protocols of MANET. The classification of protocol depends on, how the protocol handles the packet delivery from source to destination. Each protocol has its own category according to that, it works. The Routing protocol mainly classified into three categories: Proactive, Reactive and Hy $\neg$ brid protocols. [5]

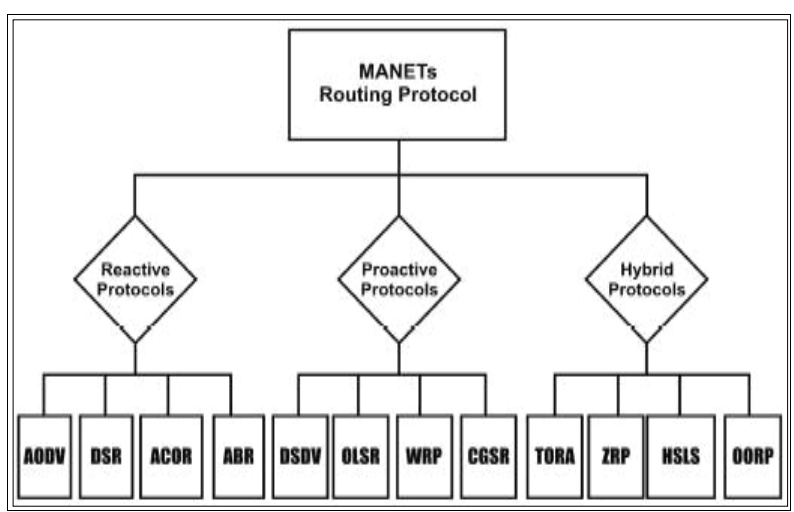

Fig 2: MANET Routing Protocols 


\subsubsection{Proactive Routing protocol}

In these types of Protocols, the route to nodes is maintained in routing table that's why it is also known as table driven protocols. In this table, the route is specified and the packets are traveled on the pre- specified route. This technique has an advantage that, the transferring of packets is done faster. But on the other hand it has also a disadvantage of routing overheads, cause of defining all the routes before transferring the packets. The OLSR (Optimized Link Stare Routing) and DSDV (Destination Sequence Distance Vector) are some examples of Proactive Protocols

\subsubsection{Reactive Routing Protocols}

In this type of protocol, the routes are not predefined for routing but they found when transmission is needed. That's why it is known as On Demand Protocol. This feature reduces the routing overheads and it is an advantage of this protocol [11].

For providing a root on need there is a mechanism works that is based on an algorithm named Flooding Algorithm. In this algorithm a node broadcasts the data packet to intermediate nodes and they forward them further and so on until it received by destination. The reactive protocols can perform pleasingly at resource limited environment.

The examples of Reactive Protocol are DSR (Dynamic Source Routing), AODV (Ad-hoc on Demand Distance Vector) and AOMDV.

\subsubsection{Hybrid Routing Protocols}

The Hybrid Protocol is a combination of Proactive and Reactive protocol approaches. Using both protocols, the advantages of both protocols are inherited. [17]

\section{AODV PROTOCOL}

The AODV is stands for Ad hoc On-Demand Distance Vector. It's a routing protocol for ad hoc networks and other wireless networks. This technique allows the wireless nodes to get routes for new destinations. This protocol establishes a route of path to destination nod only when transmission needed, so it can be called a Reactive Protocol.

The AODV Protocol allows the wireless networks to respond link changes and damages in network topology according to time limit. Some Control Messages are used in AODV which are mentioned below:

- $\quad$ Route Request Message(RREQ)

- Route Reply Message(RREP)

- $\quad$ Route Error Message(RERR)

- Route Reply Acknowledgement Message(RREPACK)

If any node wants to send a message to any destination node and it doesn't have a valid route for destination then a process is started to discover a new path which passes the message through the other related nodes and those nodes work as routers. The process of communication started with RREQ in which source node send the message to their nearest node and these node sends the message their neighbor node. The process of RREQ performs continuously, until RREQ does not received by destination. Once a request received by destination, the communication process started between sender and receiver. To refer the Response term
RREP abbreviation are used, which is the reply of request [4] [6] [9].

\subsection{Path/Link Care}

Once a communication started between two nodes then route of communication is maintained by the related nodes. It indicates the communication is being performed by the nodes. But if any reason such as movement of nodes and breaking of signals destination to source link break up then intermediate nodes indicates the sender or receiver fault occurred between communications (RERR). RREQ, RREP, RERR, RREP-ACK all the term are used to establish the connection and terminate the connection between nodes. RREP-ACK is used when the reply of message is to be sent to source, and the report of packet delivered is transmitted in the form of RREP-ACK. [7][10].

\section{LINK INTERRUPTION CAUSE OF MOBILITY}

The nature of mobile ad-hoc network is dynamic so that the mobility problems can be occurred in this network. If the nodes are in the transmission range then the communication can be done by this node but if the node outside the range then communication cannot perform by the node. The topology of ad-hoc network is dynamic so it can be changed according to movement and its communication also dynamic. The range of transmission, message delay time, and response time and error rate depends on the position of node. Mobile ad-hoc network discover all nodes that is stay in transmission range [13][17].

\subsubsection{New Route Discovery Cause of Interruption}

Link Interruption occurs when nodes move out from network range. In this case, the transmission link break between them and route discovery re-discover the network nodes. The following nodes discovered by the network:

- $\quad$ Source Node

- Intermediate Node

- Destination Node

\subsubsection{Source Node}

It is the node that sent the RREQ to another node for communication.

\subsubsection{Intermediate Node}

In the intermediate node more than one node can be group to make the communication path. These nodes work as router for communication

\subsubsection{Destination Node}

It is the node that sent the RREP of RREQ to requested node to start the communication.

The closeness of next node identified with the help of the mathematical equation that is: [14][17]

$\mathrm{DC}=(1-(\mathrm{Di} / \mathrm{Dc}))$ Here

Di: Shortest distance from neighbor node i to destination D.

Dc: Shortest distance from packet forwarding node $\mathrm{c}$ to destination D.

. The path is maintained entire the transmission period if once established. But some nodes no longer are within the scope of other nodes. Cause of this reason paths become disabled.

But, there is an option to solve this problem that is, we have to lead the process to discover the path and control packets are generated. To understand the initial set up of nodes and 
generated paths between different destination and source nodes in respective manner we have a Fig. 3 .

Di/Dc: closeness of the next hop

\section{AODV PROTOCOL IMPLEMENTATION IN NS-2}

\subsection{Performance measure link Throughput}

No. of bits denote bit per second transmitted by the both nodes, and observation duration denotes the receiving capacity of the destination node. [15]

$$
\mathrm{Y}=\frac{\text { No. of bits source to destination }}{\text { Observation duration }}
$$

\subsection{End to End Delay}

In End-to-end delay the period in which the source node sending the packet till receiving the destination node those packets. The route developing time and packets transmission time are included in it.

It is the average time in which data packets reaches to the destination. End to end delay calculated by subtracting time at which first package was transmitted by source and the time at which first data packet arrived to destination.

$\mathrm{E}[$ delay $]=\sum$ all delay samples/No. Of samples

Average delay is computed by above formula. Here delay sample is delay between packets transmission that associated with a packet.

\subsection{Delivery Ratio}

The delivery ratio is also a parameter which shows the transmission's efficiency of total amount of data and the data which is delivered by the source node. It is divide the original message into packets to increase the speed of transmission. When the entire packets received by the receiver then the packet rearrange into original form.

Here, size of packets: 1000 Bytes

Each packet breaks in 1000 byte.

\subsection{Bandwidth}

This parameter deals with the section, in which speed of the data packets to be sent to destination node. But it is more important aspect that no data should be lost during the transmission. The Bandwidth is the important aspect of network in which the speed of network can be measure easily. [8] [13]

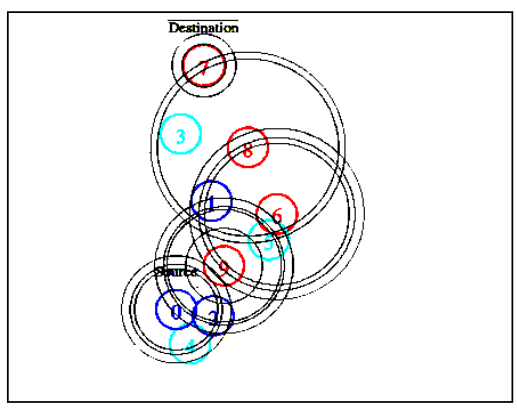

Fig 3: Network with source and Destination

The above Fig. 3 shows setup of nodes and dynamic mobility of nodes. Nodes are dynamic in the above Fig. 5 because it is an ad-hoc network and the position of nodes changed dynamically at the starting setup of nodes. The color of nodes shows the different network framework from that these are belongs. The circle in the network shows the transmission range of nodes. If any node comes in range of this circle, that can receives the signal from sending nodes. These radio waves range are used to transmit the signal to another node. This diagram shows the source to destination path and way of transmission.

Some problems arise in the ad-hoc network while transferring the data. The problem is that if the size of receiving buffer is less then transmitting buffer then packet loss problem can occurs. And also a problem, at the transmission time if node goes to outside of network range then communication fault occurred there. It is also called link break problem. The following Fig. 4 shows these circumstances [18].

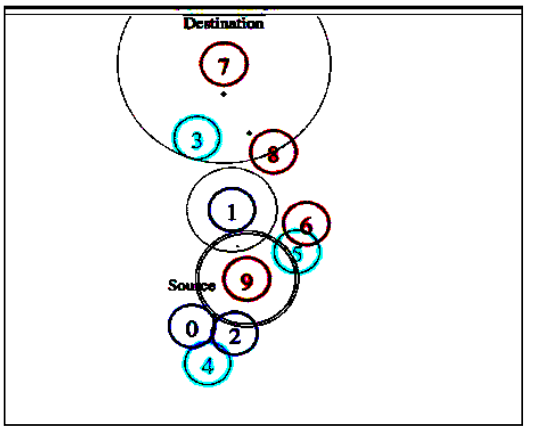

Fig 4: Link Break Mobility and Packet Drops

Table 1. Simulation Setup Parameters

\begin{tabular}{|c|c|}
\hline Network Range & $600 \times 800 \mathrm{~m}$ \\
\hline Transmission Range & $150 \mathrm{~m}$ \\
\hline No. Nodes & 10 \\
\hline Bandwidth & $2.5 \mathrm{Mbps}$ \\
\hline Traffic Type & CBR \\
\hline Packet Size & 1000 Bytes \\
\hline
\end{tabular}


Table 2. Simulation Set Up By Tcl Codes

\begin{tabular}{|c|c|c|}
\hline set val(chan) & $\begin{array}{l}\text { Channel/Wire } \\
\text { less Channel }\end{array}$ & ;\#channel type \\
\hline set val(prop) & $\begin{array}{c}\text { Propagation/ } \\
\text { TwoRayGround }\end{array}$ & ;\#radio-propagation model \\
\hline set val(netif) & $\begin{array}{c}\text { Phy/Wireless } \\
\text { Phy }\end{array}$ & $\begin{array}{c}\text {;\#network interface } \\
\text { type }\end{array}$ \\
\hline set val(mac) & Mac/802_11 & ;\#MAC type \\
\hline set val(ifq) & Queue/DropTail/PriQueue & ;\#interface queue type \\
\hline set val(ll) & $\overline{L L}$ & ;\#link layer type \\
\hline set val(ant) & Antenna/Omni Antenna & ;\#antenna model \\
\hline set val(ifqlen) & 50 & ;\#max packet inifq \\
\hline set val(nn) & 10 & ;\#number of mobile nodes \\
\hline set val(rp) & AODV & ;\#routing protocol \\
\hline set $\operatorname{val}(\mathrm{x})$ & 600 & ;\#X dimension of topography \\
\hline set val(y) & 800 & ;\#Ydimension of topography \\
\hline set val(stop) & 20 & ;\#time of simulation end \\
\hline
\end{tabular}

The Fig. 4 shows the packets dropping problem and link break cause of mobility problem. This problem can be solved by the network but for this some provisions are required to make the communication better. The transmitting node sends the data according to receiving node limit, the receiving buffer limit of nodes should be average. The way of transmission can make the better performance of network.

Nodes are dynamic in the ad-hoc network and AODV work with mobility of nodes. Nodes in the network dynamically moved the next figure showing this condition in the network where nodes can be moved at the time of transmission. And also it is performed continuously route discovery process. This process scans the entire device which is currently present in the network. We have a figure that shows this condition

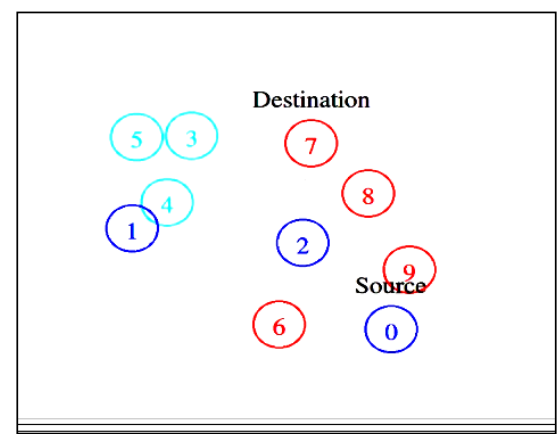

Fig 5: Mobility of Nodes and Route Discovery
The above Fig. 5 shows the route discovery process in the network and mobility of nodes that changed dynamically. Nodes find the shortest path to send the data in minimum time it is the advantage of network. If a network have two path shortest and longest then it will sends the data from shortest path to reduce the delay time. Movement of path dynamically, changed by the node. But if node moves to outside the network then it tries to make the connection in some situation. [16]

Intermediate nodes perform an important role in this simulation network in which these are responsible for dynamic interchange of data. It has performed the role as router in the network and transmit the data continuously one node another until it reaches at destination. The next section deals with related figure.

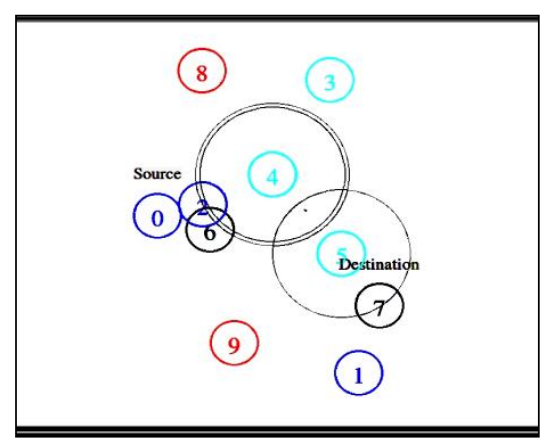

Fig 6: Intermediate Nodes Involvement in Transmission 
The above figure shows that, the Intermediate node works between the source node and destination nodes. Here we indicate the intermediate nodes range in the circle form. The spreading of circle indicates the area of transmitting. All the transmission is done by the intermediate nodes. These intermediate nodes work as a router between the source and destination nodes.

In this transmission, if there is more difference between source node and destination node and there is no any other network between them then the data transmission is not possible without intermediate node. If the delay time decrease and respond time increases then the performance of network may be much better. Below graph shows rate of transmission.

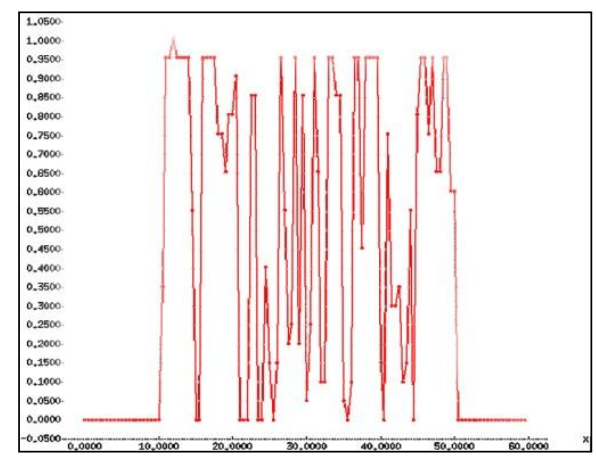

Fig 7: Rate of Transmission

The above Fig. 7 shows the flow of data transmission in the form of graph here we illustrate the bit rate and bandwidth of network according to speed and circumstances of network. Here some lines heights are longer and some are smaller. The graph also showing the delay time of network in which it has respond. The dotted line is showing the speed maintainability ratio. Some positions of the line in the graph are equal that is showing the equality bandwidth rate of transmission.

Packet delivery ratio is an aspect of communication in which the packet transmission depends on end to end delay. It means if a node requested to another node then it will waiting for response of another node. End to end delay is the time between one node to another by which request and response sends in how much time to perform the task.

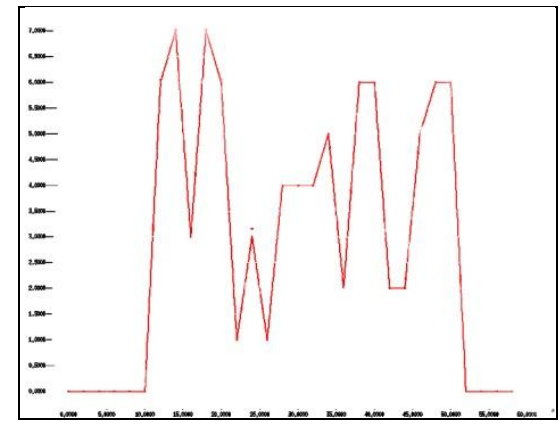

Fig 8: End-to-end delay and Packet delivery ratio

The Fig. 8 shows the end to end delay and packet delivery ratio graph in which the rate of packet transmission depends on end user response. If the response time is longer, then the speeds of transmission slow down. But if the twining between both nodes is perfect then the resulted throughput increased. This aspect is depends on overall involved simulation devices. So the communication can perform faster.

\section{CONCLUSION AND THE FUTURE EXTENSION}

The AODV as a reactive routing protocol includes AODV related aspects which illustrate the need of network and how the transmission performed in this network. This Protocol can be used in future to get the proper network transmission and ease to virtualize the network. Using this protocol the performance of network can be increased and this paper shows that AODV protocol increases the throughput of the network.

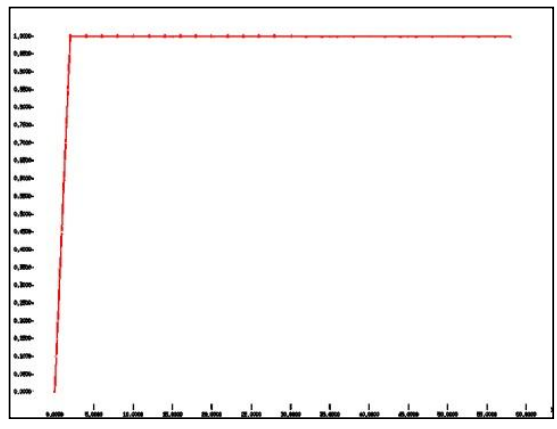

Fig 9: Throughput Improvement

\section{REFERENCES}

[1] Term "Back pressure", "The Multi-hop Queuing Network Model", source from Wikipedia

[2] jiang wu, "a simulation study on using the virtual node Layer to implement efficient and reliable manet Protocols" chapter 7 pp.149-152, 2011

[3] Term "Mobile Adhoc network", "Types of MANET", source from Wikipedia

[4] Term "Network Simulator", "NS2", source from isi.edu

[5] P. Manickam, T. GuruBhaskar, M. Girija and Dr. D. Manimegalai, "performance comparisons of routing Protocols in mobile ad hoc networks" International Journal of Wireless \& Mobile Networks (IJWMN) Vol. 3, No. 1, February 2011

[6] Term "Reactive Protocols", "Control message for route maintenance", source from olsr.org

[7] UMI ,umi no. 3301521.Copyright 2008 by ProQuest Information and Learning Company, Ann, Arbo. Chapter 2, PgNo.5.

[8] Term "MANET Routing protocols Performance", "Route Discovery, Packet delivery, End to end delay, Throughput", source from intechopen.com

[9] Samyat Shah, Amit Khandre, Mahesh Shirole and Girish Bhole, "Performance Evaluation of Ad Hoc Routing Protocols Using NS2 Simulation", unpublished

[10] Shweta Modi, Jitendra Prithviraj, "Performance Comparison of IACO,AODV Networking Routing Protocols", International Journal of Smart Sensors and Ad Hoc Networks (IJSSAN), 2011.

[11] Parviner Singh, Dinesh Singh, Vikram Singh, "Evaluation of Routing Protocols in manets with Varying Network Scope", 2012 International Conference on Information and Network Technology (ICINT 2012) IPCSIT vol. 37 (2012) (C) (2012) IACSIT Press, Singapore. 
[12] Term "MANET Message Format", "Message Format", source from eurasipjournals.com

[13] Term "Delay Calculation in ns2", "End to end delay, delay computation", source from ns2ultimate.com

[14] K. Prasant, K. Duraiswamy, "Minimizing End to End Delay in vanets using Potential Edge Node Based Greedy Routing Approach", European Journal of Scientific Research ISSN 1450-216X Vol.48 No.4 (2011), pp.631647 ( ) eurojournals Publishing, Inc. 2011.

[15] Term "Throughput Calculation in ns2", "Performance measure link throughput", source from ns2ultimate.com

[16] Isaac Woungang, Sudip Misra, Subhash Chandra Misra,"Guide to wireless ad-hoc networks",European
Journal of Scientific Research ISSN 1450-216X Vol.48 No.4 (2011), pp.631-647@ eurojournals Publishing, Inc. 2011, Springer-Verlag London Limited 2009. Chapter 6, PgNo. 77

[17] Lavneet Bansal, Swati Jindal, "Performance Analysis and Comparison of Reactive And Proactive MANET Routing Protocols", International Journal of Computer Applications (0975 - 8887) Volume 30- No.8, September 2011.

[18] Soufiene Djahel, Secure Routing And Medium Access Protocols in wireless Multihop networks, GRIN, ISBN 978-3-656-04102-3. Chaptor2, PageNo.8. 